\title{
An LBM based model for initial stenosis development in the carotid artery
}

\author{
A.C. Stamou \& J.M. Buick \\ School of Engineering, Anglesea Building, Anglesea Road University of Portsmouth, \\ Portsmouth, PO1 3DJ, UK \\ E-mail: James.Buick@port.ac.uk
}

\begin{abstract}
A numerical scheme is proposed to simulate the early stages of stenosis development based on the properties of blood flow in the carotid artery, computed using the lattice Boltzmann method. The model is developed on the premise, supported by evidence from the literature, that the stenosis develops in regions of low velocity and low wall shear stress. The model is based on two spatial parameters which relate to the extent to which the stenosis can grow in each development phase. Simulations of stenosis development are presented for a range of the spacial parameters to determine suitable ranges for their application. Flow fields are also presented which indicate that the stenosis is developing in a realistic manner, providing evidence that stenosis development is indeed influenced by the low shear stress, rather than occurring in such areas coincidentally.
\end{abstract}

Keywords: Stenosis modelling, Blood flow modelling, Atherosclerosis, lattice Boltzmann method

Accepted for publication in Journal of Physics A: Mathematical and Theoretical

\section{Introduction}

Atherosclerosis is one of the major causes of ischemic stroke as it can lead to the narrowing of blood vessels due to a build-up of plaque, and is a major cause of fatality each year [1]. There is a growing body of evidence which suggests a correlation between regions of low near-wall velocity and low Wall Shear Stress (WSS), and early development of atherosclerosis [2-7]. It has also been suggested that, within such regions, oscillatory flow may also be associated with regions where stenosis is observed to develop [8-10]. Here we consider a model for the early stages of stenosis development to provide a tool for studying both the developing stenosis geometry, and how this affects the blood flow in the artery. The model is focused on the carotid artery and the Lattice Boltzmann Model (LBM) is used to simulate the blood flow. 
Arterial flows are commonly studied using Computational Fluid Dynamics (CFD). The LBM is a relatively novel technique to simulating fluid flow and is based on a mesoscopic kinetic approach. This approach has been validated both theoretically, where it can be shown that it mimics the Navier-Stokes equation up to second order, and through its application to a range of diverse flow problems including acoustically induced flows [11], bubble dynamics [12], free surface flows [13] and flow in porous media [14]; as well as a range of blood flow simulations [15-19].

LBM simulations have been considered where the flow of the blood affects a change in the boundary geometry. This has been applied [20-24] for blood clotting, either on a backward facing step or a pre-existing idealised stenosis; and [25] for the development of a stenosis in an originally healthy artery.

Tamagawa and Matsuo [20] considered a model for blood clotting based on tracer particles added to the flow. These particles were transported by the flow and adhered to the wall when both the shear rate and the distance of the particle from the wall were below individual thresholds. This work was extended [21] where concentration and surface tension were also considered. An alternative approach by $[22,23]$ considered blood clotting based on fluid residence time. Fluid nodes where the tracer particles reached a prescribed 'clotting time' were converted to wall nodes. Moiseyev and BarYoseph [24] introduced a Virtual Particle Integration (VPI) method [26] to calculate real time integrals of the stress. Coagulation of the blood was modelled to occur when the VPI stress accumulation and a residential time, similar to that considered by [22], exceeded a pre-determined threshold.

Karimpour and Javdan [25] considered an LBM model based on the Oscillatory Shear Index (OSI) calculated along the artery wall. Regions of high OSI were deemed to be prone to atherosclerosis and layers of deposits were progressively deposited in these regions. Although no algorithm is presented, this appears to be based on a predetermined threshold similar to [20-23] and results in a layer of deposit at each step. The authors identify difficulties in applying their strategy due to the step-like nature of their artery walls which lead to boundary roughness; difficulty in defining the wall normal; and fluctuations in the wall velocity which feed into the OSI calculation. To address this the authors introduced a 'parallel' surface three units from the wall which was used to calculate the WSS. The artery normal was defined as the normal to this surface.

Here we consider an LBM based model for the development of an arterial stenosis. Following [25] this is performed in 2D and a rigid wall approximation is applied. Although artery walls are compliant and the difference between the systolic and diastolic diameter is around $10 \%$ [27] for a health artery; in stenosed regions the walls become ridged. Given that the focus here is on stenosis development, the rigid wall approximation was considered to be appropriate. The model developed here uses an extrapolation boundary condition [28] which enables the geometry of the wall to be simulated to a sub-grid accuracy. This is important, not only because it removes the step-like geometry and produces a smooth velocity and shear profile at the wall, 
but also because it allows a truly microscopic model to be developed. Additionally the stenosis development does not rely on arbitrary thresholds and is based on the local WSS and near-wall velocity which are generally considered to be the primary flow variables correlated with stenosis.

\subsection{The LBM model}

The D2Q9 LBM evolves according to the kinetic equation

$$
f_{i}\left(\boldsymbol{x}+\boldsymbol{e}_{i}, t+1\right)-f_{i}(\boldsymbol{x}, t)=C_{i}
$$

where $i=0,1, \ldots, 8$ labels the link directions of the underlying grid:

$$
\begin{array}{ll}
\boldsymbol{e}_{0}=(0,0) & \\
\boldsymbol{e}_{i}=\left(\cos \left(\frac{\pi}{2}(i-1)\right), \sin \left(\frac{\pi}{2}(i-1)\right)\right) & \text { for } i=1,2,3,4 \\
\boldsymbol{e}_{i}=\sqrt{2}\left(\cos \left(\frac{\pi}{2}(i-1)+\frac{\pi}{4}\right), \sin \left(\frac{\pi}{2}(i-1)+\frac{\pi}{4}\right)\right) & \text { for } i=5,6,7,8,
\end{array}
$$

and $f_{i}$ denotes the distribution function for each of the discrete link directions. Equation (1) represents two processes. Firstly the LHS involves streaming of the distribution functions along the links from the fluid node $\boldsymbol{x}$ to the neighbour at $\boldsymbol{x}+\boldsymbol{e}_{i}$. The RHS of equation (1) is the collision function which mimics collisions between the distribution functions.

The velocity and density of a fluid described by equation (1) are determined locally at each fluid node from the distribution functions as

$$
\rho(\boldsymbol{x}, t)=\sum_{i=0}^{i=8} f_{i}(\boldsymbol{x}, t) \quad \text { and } \quad \rho(\boldsymbol{x}, t) \boldsymbol{u}(\boldsymbol{x}, t)=\sum_{i=0}^{i=8} f_{i}(\boldsymbol{x}, t) \boldsymbol{e}_{i} .
$$

The collision operator $C_{i}$ is given by the Bhatnagar-Gross-Krook approximation as $[29-31]$

$$
C_{i}=-\Omega\left[f_{i}(\boldsymbol{x}, t)-f_{i}^{e q}(\boldsymbol{x}, t)\right],
$$

where $\Omega$ is the relaxation frequency and $f_{i}^{e q}(\boldsymbol{x}, t)$ is the equilibrium distribution function:

$$
f_{i}^{e q}(\boldsymbol{x}, t)=w_{i} \rho\left(1+3 \boldsymbol{e}_{i} \cdot \boldsymbol{u}+\frac{9}{2}\left(\boldsymbol{e}_{i} \cdot \boldsymbol{u}\right)^{2}-\frac{3}{2} \boldsymbol{u}^{2}\right),
$$

where $w_{0}=4 / 9, w_{1}=w_{2}=w_{3}=w_{4}=1 / 9$ and $w_{5}=w_{6}=w_{7}=w_{8}=1 / 36$. Combining equations (1) and (4), expanding in terms of the Knudsen number [32], and applying a Chapman-Enskog expansion [33] yields the mass and momentum equations in the incompressible limit [34]

$$
\frac{\partial \rho}{\partial t}+\rho \nabla_{\alpha} u_{\alpha}=0
$$

and

$$
\frac{\partial \rho u_{\alpha}}{\partial t}+\nabla_{\beta} \Pi_{\alpha \beta}=0
$$

where

$$
\Pi_{\alpha \beta}=\rho u_{\alpha} u_{\beta}+p \delta_{\alpha \beta}-2 \nu \rho S_{\alpha \beta},
$$




$$
\begin{aligned}
& \nu=\frac{2-\Omega}{6 \Omega} \\
& S_{\alpha \beta}=\frac{1}{2}\left(\frac{\partial \rho u_{\alpha}}{\partial_{\beta}}+\frac{\partial \rho u_{\beta}}{\partial_{\alpha}}\right)
\end{aligned}
$$

and

$$
p=c_{s}^{2} \rho
$$

with $c_{s}=1 / \sqrt{3}$. It can be shown [35] that the strain rate tensor, $S_{\alpha \beta}$, can be calculated locally at each LBM node as

$$
S_{\alpha \beta}=\frac{-3 \Omega}{2} \sum_{i}\left(f_{i}-f^{e q}\right) e_{i \alpha} e_{i \beta} .
$$

Roman subscripts are used here as labels while Greek subscripts are used to represent vector components and summation over repeated Greek indices is assumed.

\subsection{Boundary Conditions}

The artery geometry is defined by a series of boundary points $\boldsymbol{b}_{j}$ which lie at the intersection of the artery wall and each link which cuts it. Boundary conditions are applied at each boundary point $\boldsymbol{b}_{j}$ using a sub-grid accurate extrapolation boundary scheme [28] which maintains the second-order accuracy of the LBM while describing the geometry of the artery to a resolution greater than the underlying lattice [36,37]. The pulsatile nature of the blood flow was introduced at the base of the artery using the waveform adapted from [38] and an extrapolation method [39] is applied at the outflow. The artery geometry and the cardiac pulse have been applied previously $[37,40]$ and are presented in section 3 .

\section{Stenosis Growth Model}

Evidence from the literature indicates that stenosis development occurs in regions with low wall shear stress and low near-wall velocity [2-7]. Here we describe a model to simulate the growth of the stenosis based on the details of the blood flow.

For each period the position for the stenosis growth is identified. This is selected from the nodes $\boldsymbol{x}_{j}^{B}$ which are the sub-set of all wet nodes which have one or more links passing through a boundary site; that is they are adjacent to the artery wall. For each wall-adjacent node a grid-based normal $\boldsymbol{n}_{j}$ is also defined based on one of the $k$ links $\boldsymbol{e}_{i}^{B}$ which intersect the boundary. When $k=1$, (only one link, $\boldsymbol{e}_{1}^{B}$, cuts the artery wall) the normal is set to $-\boldsymbol{e}_{1}^{B}$. The negative sign is required to give an outward pointing normal. For $k>1$ a vector $\boldsymbol{B}$, approximating the local boundary, is defined joining the first and last boundary point associated with the node. For example, in figure 1 the hexagonal node has two links $\boldsymbol{e}_{1}^{B}=\boldsymbol{e}_{3}$ and $\boldsymbol{e}_{2}^{B}=\boldsymbol{e}_{6}$ which cut the boundary (thin solid line) at the boundary points (solid squares) $\boldsymbol{b}_{0}$ and $\boldsymbol{b}_{1}$ and so $\boldsymbol{B}=\boldsymbol{b}_{0}-\boldsymbol{b}_{1}$ and $\boldsymbol{n}_{j}=-\boldsymbol{e}_{3}$ is the 

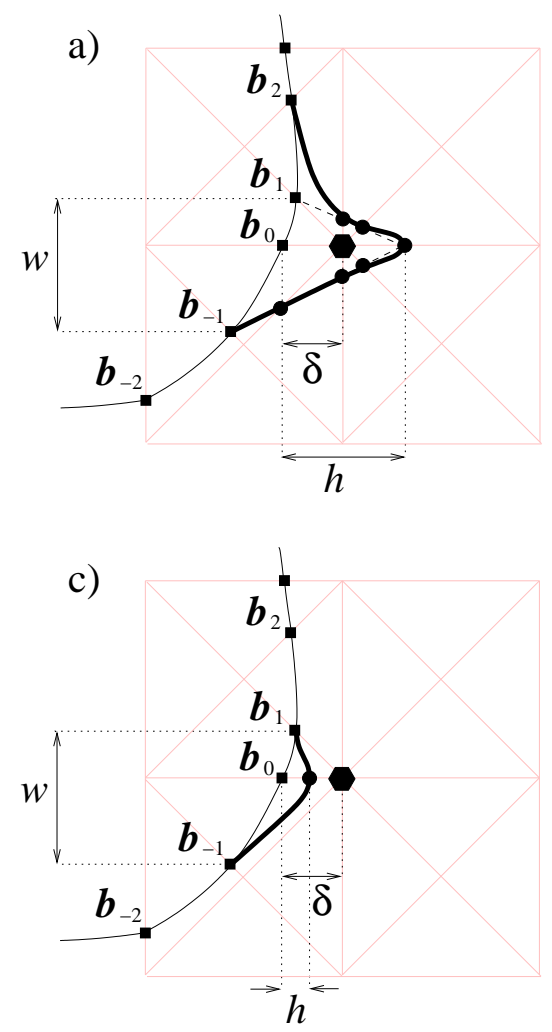

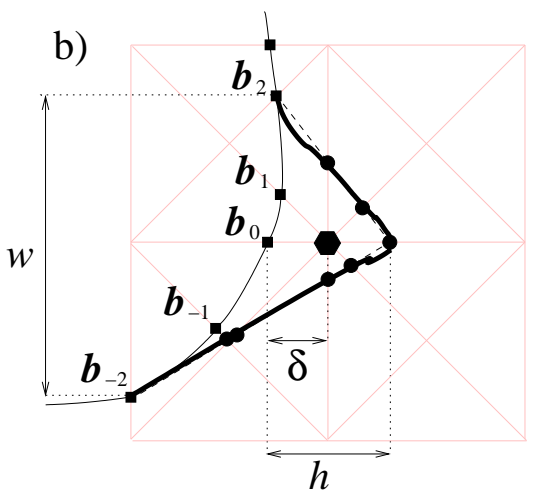

- Selected fluid node

- Original boundary point

- New boundary point Original boundary New boundary

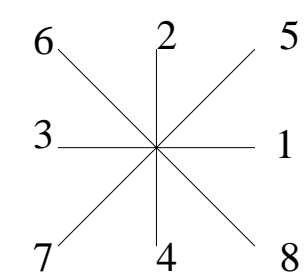

Figure 1. Model for the wall development is shown for selected values of $h$ and $w$.

closest grid-based normal. This is determined numerically as the link which minimises

$$
\frac{\left|\boldsymbol{e}_{i}^{B} \cdot \boldsymbol{B}\right|}{\left|\boldsymbol{e}_{i}^{B}\right||\boldsymbol{B}|} .
$$

The position where stenosis development will be modelled is determined from the near-wall haemodynamics over a period of motion. The velocity and $S_{\alpha \beta}$ are known at each of the nodes $\boldsymbol{x}_{j}^{B}$; however, it is not appropriate to use these values since the nodes are at varying distances from the wall. Following the methodology of the extrapolation boundary scheme [28], the velocity is calculated a fixed distance of 1 lattice unit (lu) from the wall, along the direction $\boldsymbol{n}_{j}$. This can be found as an extrapolation (or an interpolation for the case $\delta>1$, which can occur on a diagonal link):

$$
\boldsymbol{u}^{(1)}\left(\boldsymbol{x}_{j}^{B}, t\right)=\frac{\boldsymbol{u}\left(\boldsymbol{x}_{j}^{B}, t\right)}{\delta}
$$

where $\delta$ is the distance along $\boldsymbol{n}_{j}$ between the node and the boundary point, as illustrated in figure 2. Note that $\boldsymbol{u}^{(1)}\left(\boldsymbol{x}_{j}^{B}, t\right)$ is not the velocity at position $\boldsymbol{x}_{j}^{B}$, it is the velocity associated with node $\boldsymbol{x}_{j}^{B}$, but evaluated a distance of 1 lu from the boundary. An alternative expression, see figure 2 , is given by

$$
\boldsymbol{u}^{(1)}\left(\boldsymbol{x}_{j}^{B}, t\right)=\frac{(1-\delta) \boldsymbol{u}\left(\boldsymbol{x}_{j}^{B}+\boldsymbol{n}_{j}, t\right)+\left(n_{j}-1+\delta\right), \boldsymbol{u}\left(\boldsymbol{x}_{j}^{B}, t\right)}{n_{j}}
$$




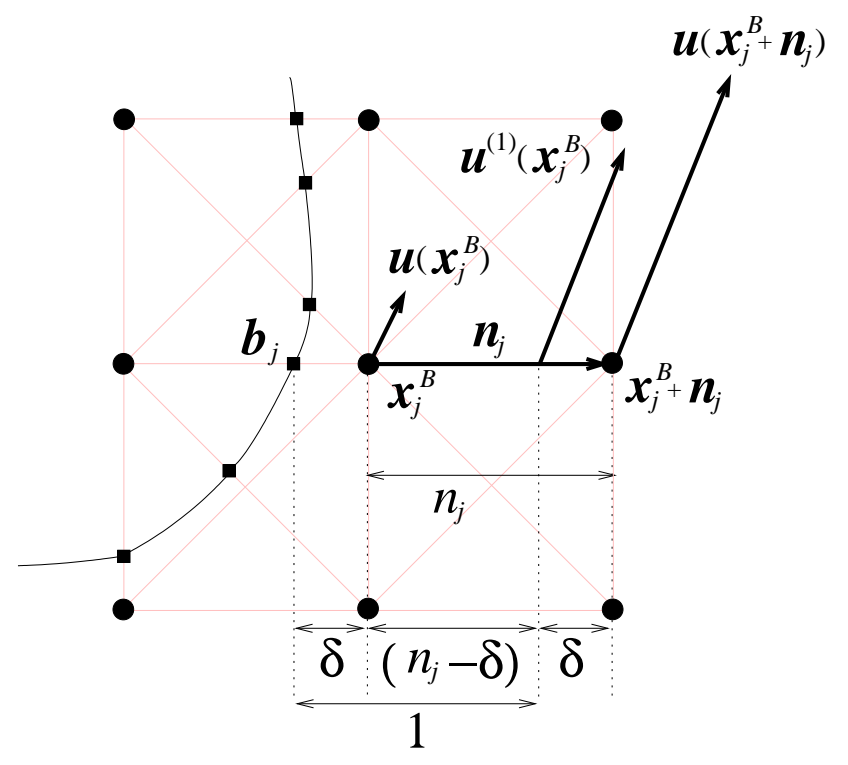

Figure 2. The near-wall velocity $1 \mathrm{lu}$ from the wall for a single value of $j$.

where $n_{j}=\left|\boldsymbol{n}_{j}\right|$ is 1 when the normal is horizontal or vertical, as shown in figure 2, and $\sqrt{2}$ when the normal is diagonal.

In a similar way, the value of $S_{\alpha \beta}\left(\boldsymbol{b}_{j}, t\right)$ at the wall can be found as

$$
S_{\alpha \beta}\left(\boldsymbol{b}_{j}, t\right)=\left(1+\frac{\delta}{n_{j}}\right) S_{\alpha \beta}\left(\boldsymbol{x}_{j}^{B}, t\right)-\frac{\delta}{n_{j}} S_{\alpha \beta}\left(\boldsymbol{x}_{j}^{B}+\boldsymbol{n}_{j}, t\right),
$$

where $S_{\alpha \beta}\left(\boldsymbol{x}_{j}^{B}, t\right)$ and $S_{\alpha \beta}\left(\boldsymbol{x}_{j}^{B}+\boldsymbol{n}_{j}, t\right)$ are calculated directly from the distribution functions using equation (12).

Thus the near-wall haemodynamics can be described in terms of the near-wall velocity using equations (14) or (15) and the WSS through equation (16). In practice it was found that the regions of low near-wall velocity magnitude were consistent with the regions of low WSS magnitude. This can be understood since close to the boundary the flow will be in the tangential direction, then from equation (14), $\left|\boldsymbol{u}^{(1)}\right|=|\boldsymbol{u}| / \delta$ is proportional to the wall shear stress. Although the selection of $1 \mathrm{lu}$ is arbitrary for calculating the near-wall velocity it has been shown to be a useful approach [40] to give a good measure of the velocity close to the wall. Thus to incorporate both the concept of low near-wall velocity and low WSS, the position where the stenosis development will be modelled for the $n$th period is selected as the node $\boldsymbol{x}^{B}$ with the minimum value of

$$
\int_{n \tau}^{(n+1) \tau} \frac{\left|\boldsymbol{u}^{(1)}\left(\boldsymbol{x}^{B}, t\right)\right|}{\tau} \mathrm{d} t,
$$

where $\tau$ is the length of the period. To prevent numerical instability for small $\delta$, equation (15) is used in preference to equation (14).

We note that the link based normal will be at an angle to the true normal at the boundary point. This introduces a small level of noise in the $\boldsymbol{u}^{(1)}$ profile; however the position of the minimum can still be detected and this approach was found to be 
satisfactory. Using an off-link normal is computationally more expensive and, as well as introducing additional interpolation, it also requires an approach to find the normal which will its self introduce some approximation. Two alternative approached which have been used are to consider an 'average' over a section of the artery [41], or to assume that $\boldsymbol{u}\left(\boldsymbol{x}_{j}^{B}, t\right)$ is tangential such that $\boldsymbol{u}\left(\boldsymbol{x}_{j}^{B}, t\right) \cdot \boldsymbol{n}\left(\boldsymbol{x}_{j}^{B}, t\right)=0$ [42].

\subsection{Boundary Movement}

The mechanism for modifying the artery wall, to represent the development of the stenosis, is shown in figure 1 where the hexagon represents the wet node selected using equation (17). The re-positioning of the wall is based on two parameters $h$ and $w$ which represent the height and width of the deposited plaque, as shown in figure 1. The original artery wall is shown by the thin solid line with the wet nodes (blood flow region) to the right. The boundary points, $\boldsymbol{b}_{j}$, are shown by the solid squares at the intersections of the artery wall and the grid links. For this configuration of the artery wall and the selected wet node, the normal direction is defined by equation (13) to be $\boldsymbol{e}_{1}$ and the boundary point on this link is labelled $\boldsymbol{b}_{0}$. The associated value of $\delta$ is 0.3 . The remaining boundary nodes are then labelled $\boldsymbol{b}_{ \pm 1}, \boldsymbol{b}_{ \pm 2}, \ldots$ so that the boundary points are labelled sequentially along the artery wall. Three cases are shown in figure 1 corresponding to (a) $h=0.5, w=1$, (b) $h=0.5, w=2$ and (c) $h=0.15, w=1$. For these parameters the new section of the artery wall is shown by the thick solid line and the corresponding new wall points by the filled circles. Construction lines, used to determine the new wall position, are shown by dashed lines which are often obscured by the new artery wall. In figure 1 (a) $h=0.5$ and boundary point $\boldsymbol{b}_{0}$ moves $h(0.5)$ units along the normal link $\left(\boldsymbol{e}_{1}\right)$ towards the selected wet node. Since here $h>\delta$ the boundary encapsulates the selected wet node which becomes dry. Construction lines are then drawn between this new boundary point and the old boundary links $\boldsymbol{b}_{ \pm w}$ (here $w=1$ ). New boundary points are selected where the construction lines cross the links. In figure 1 (a) the new boundary follows the lower construction line through the new boundary points. However, boundary point $\boldsymbol{b}_{1}$ is on link $\boldsymbol{e}_{6}$ out of the selected wet node. Since this is now a dry node and the link joins two dry nodes, it is not possible for it to have an associated boundary point. Thus the upper part of the new boundary connects to the next-nearest boundary point: $\boldsymbol{b}_{2}$. In figure 1 (b) $w=2$ and the construction lines connect to the old boundary points $\boldsymbol{b}_{ \pm 2}$ and the new boundary follows the construction lines. In figure 1 (c) $h<\delta$ so the selected node remains wet and the new boundary connects to $\boldsymbol{b}_{ \pm 1}$. In the event that $h=\delta$, we replace $h$ with $h-0.05$.

\section{Results}

The approach outlined in section 2.1, depends on $h$ and $w$ which have no physical significance. Thus it is important to establish how they affect the stenosis development. This scheme allows for the stenosis to develop at the end of every period; clearly this 
is not realistic. There are two very different time-scales at play: the period of the heartbeat; and the development time of the stenosis. The concept of this model is not that the stenosis develops every period; rather the assumption is that there are a large number of periods between each sequential change in the boundary and the flow properties do not change over this time. Thus there is no advantage in simulating all the intermediate periods which are identical. Based on the assumption that we only need to simulate selected periods where the stenosis changes, the number of periods will not give a precise time scale for the development of the stenosis, but rather will indicate its sequential development. Additionally, it is not evident that the relationship between the two time-scales will be linear. It is also evident that the stenosis will develop more quickly for larger values of $h$ and $w$ and so the number of periods simulated will not be a good measure of the progression since it is dependent on two non-physical parameters. For this reason, as well as investigating the effect of $h$ and $w$ on the stenosis development, it is also important to find a variable which will describe the evolution in a manner which is independent of $h$ and $w$.

The manner of stenosis development in shown in figure 3, where $T$ represents the

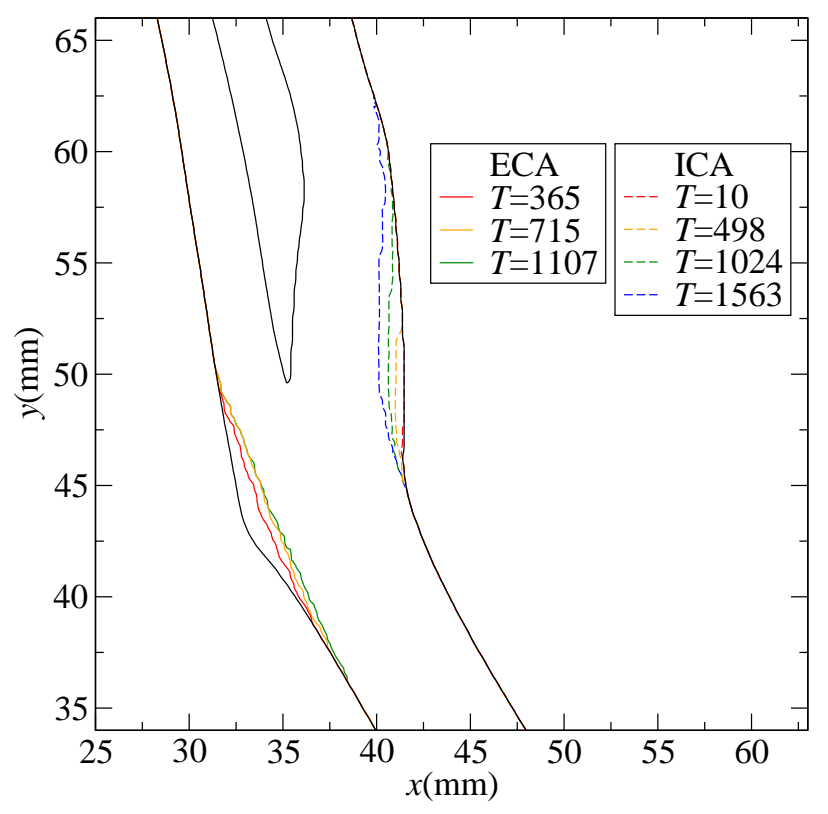

Figure 3. The development of the stenosis for $h=0.7$ and $w=1$. The stenosis geometry is shown at selected times corresponding to the final period during which each of the layer is developed.

number of periods of simulation. The development generally occurs in a series of layers which build up from the bottom towards the top of the artery. After each layer is completed a new layer starts, either on the same artery wall or on the opposite wall. For selected layers, figure 3 displays the geometry of the stenosis at the final period over which the layer develops. The layers selected in figure 3 correspond to where a switch occurs between the Internal Carotid Artery (ICA), on the right of the image, 
and the External Carotid Artery (ECA), shown on the left. Typically a larger layer, such as the one shown at $T=365$, which is formed between $T=10$ and $T=365$ is made of a number of sub-layers. While this description of the stenosis development describes the general process, there are a small number of times where this pattern is broken. In these cases the node selected by equation (17) is either on the opposite wall or at a different position on the same wall. This can occur for a few periods before it returns to the current layer. We also note that the development of these layers occurs only on the outer walls of both the ICA and ECA. Although the geometry of a stenosis can vary significantly from patient to patient, the geometry shown in figure 3 has geometrical similarities to some imaged stenosed arteries [43-46], suggesting that the model is performing in a realistic manner.

The results in figure 4 show the stenosis development for different values of $h$ in terms of $T_{s}^{*}$ when $w=1 . T_{s}$ is a sequential label indicating the number of nodes which have been converted from wet to dry. $T_{s}^{*}$ is the value of $T_{s}$ normalised with respect the minimum of the run lengths (in periods) over the range of $h$ considered. This gives a normalisation with respect to the volumetric (areal) development of the stenosis. To enable a direct comparison, only values of $T_{s}^{*}<1$ are shown in figure 4 .

The results in figure 4 show similar stenosis progression for $h=0.1,0.3$ and 0.5. The results for $h=0.7$ are similar while those for the larger values of $h$ are somewhat different in terms of the wall plaque extending less far along the wall, but further into the interior of the artery. This can be understood in terms of the mechanics of the model. When $h=1$, as soon as a wet node is selected by equation (17) it is removed from the artery and the stenosis develops into the artery by 1 lu. If $h=0.1$, then the same wet can potentially be re-selected for a further nine periods (possibly sequentially) before it is converted to a dry node. However, during this time, it is feasible that the small increments can sufficiently change the flow such that the development moves to another node; and the original one is not returned to (at least until a later stage in the development of the stenosis). Based on this picture of the stenosis development, it is evident that as $h$ increases, it will reach a level at which the resolution of the artery refinement becomes too large and the value of $h$ becomes significant in determining the evolution. Figure 4 suggests that this happens for values of $h$ between 0.5 and 0.7 . This would also suggest that the limiting value of $h$ is based on the fraction of a lattice unit, rather than the physical dimensions of $h$, e.g. as a fraction of the diameter.

To better determine the cut-off value of $h$ the value of $\Delta T_{s}^{*}$ is shown in figure 5 . This is calculated as the difference between the value of $T_{s}^{*}$ found for $h=0.5$ and that for $h=0.1,0.3,0.7,0.9$ and 1.0 in figures 5 (a) - (e) respectively. Values are shown only for nodes which were originally wet and which have been converted to dry nodes in both simulations. While the differences are generally small in figures 5 (a) and (b), there are a number of larger values. These are due to the stenosis developing in layers. There will be a number of nodes close to the edge of each layers which will, at the end of the development of the layer, be wet for one value of $h$ and dry for another - even when the difference between the geometry of the layer is so small as to have an insignificant 


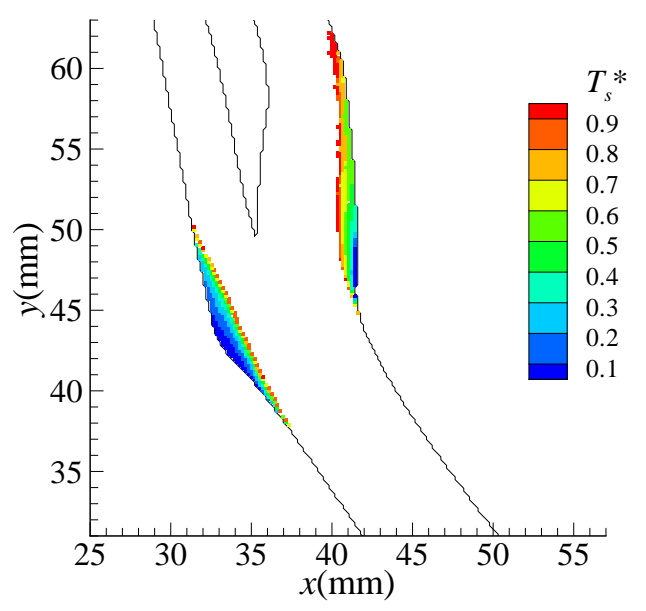

(a) $h=0.1$

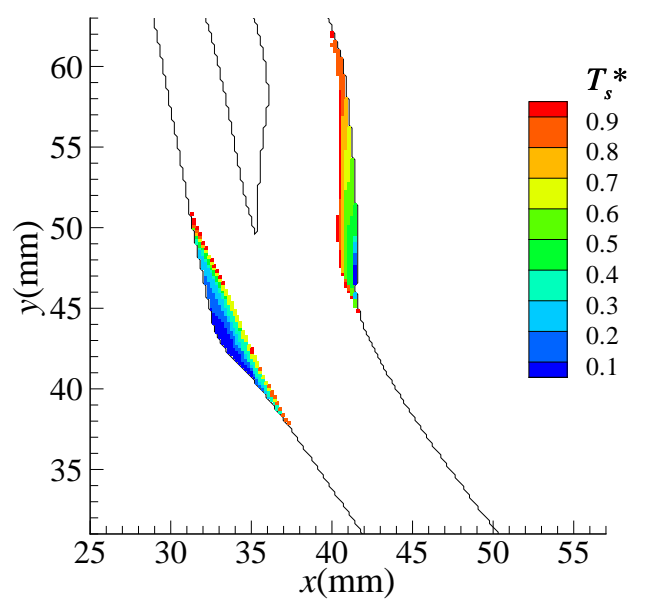

(c) $h=0.5$

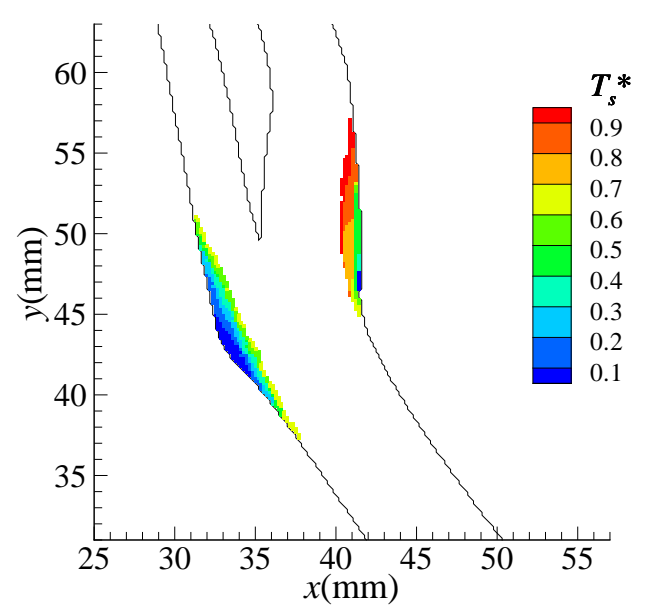

(e) $h=0.9$

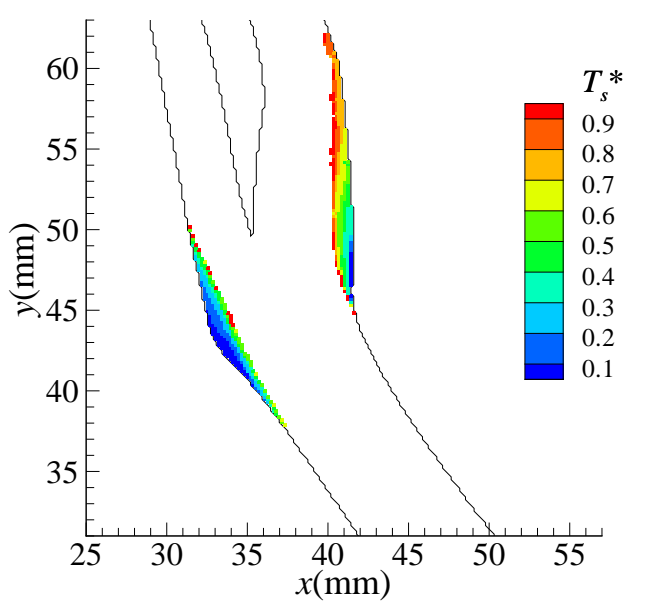

(b) $h=0.3$

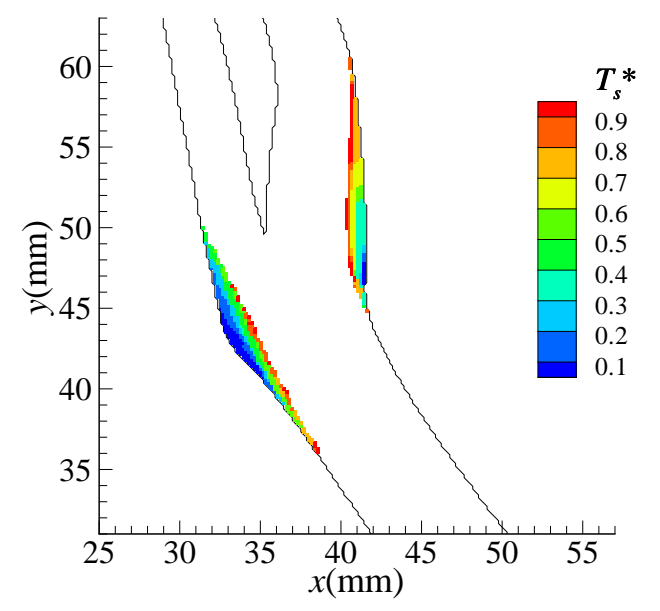

(d) $h=0.7$

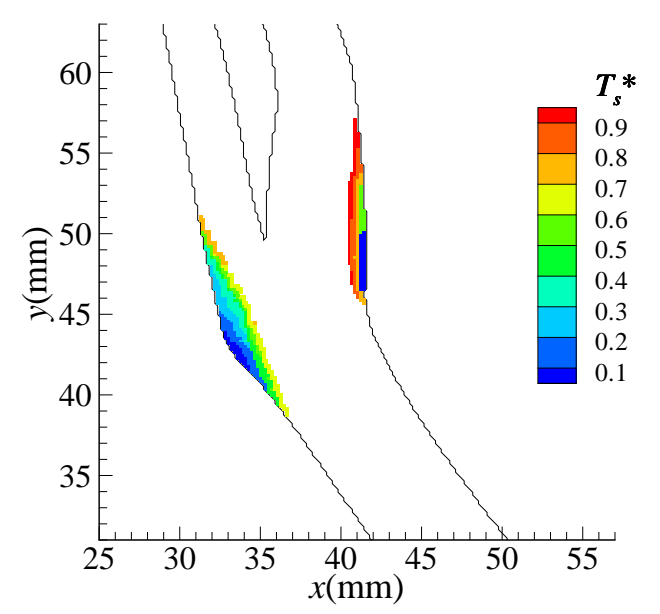

(f) $h=1.0$

Figure 4. Stenosis development in terms of $T_{s}^{*}$ for different values of $h$ when $w=1$. 


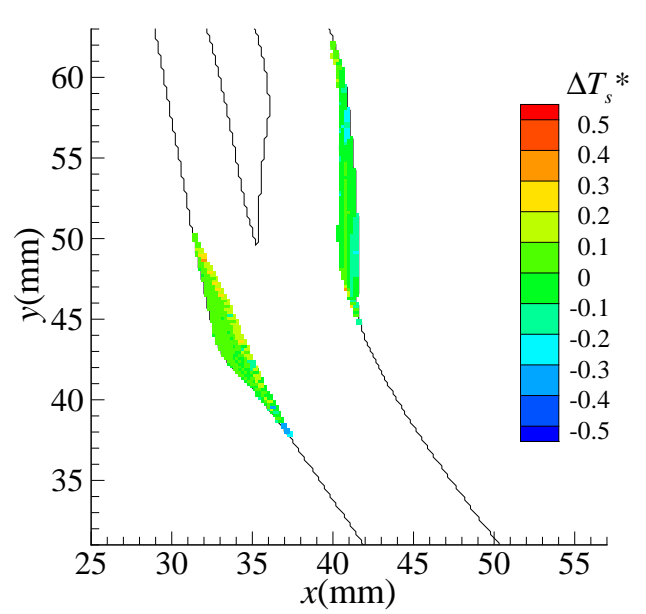

(a) $h=0.1$

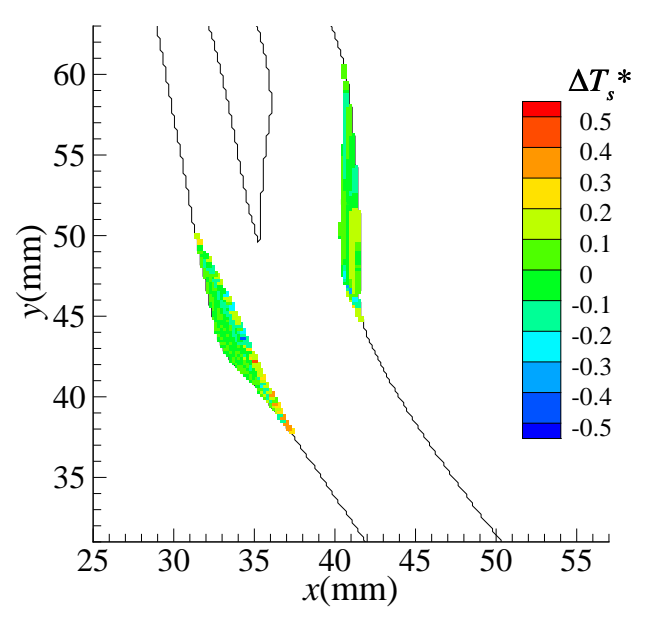

(c) $h=0.7$

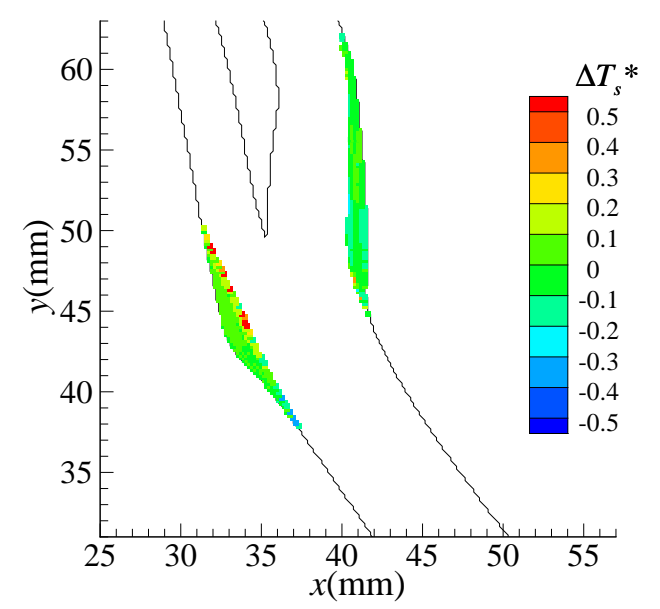

(b) $h=0.3$

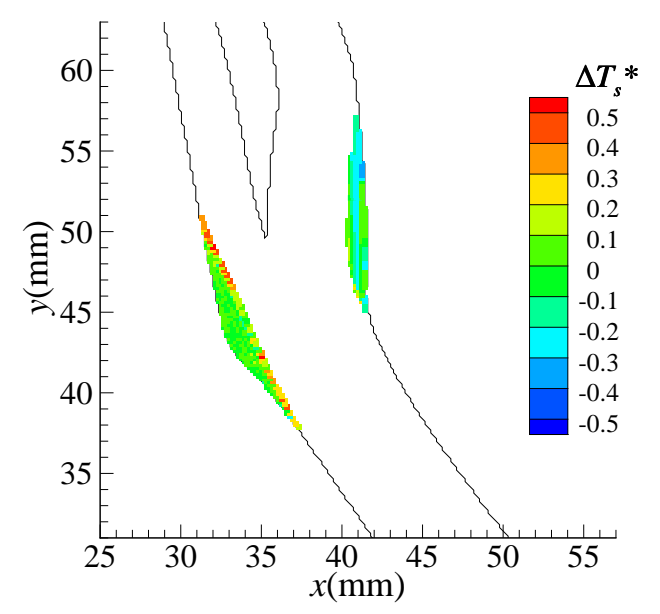

(d) $h=0.9$

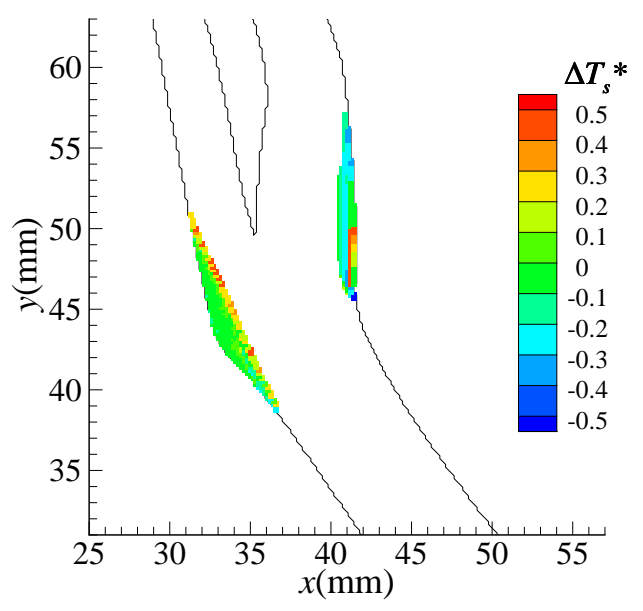

(e) $h=1.0$

Figure 5. $\Delta T_{s}^{*}$ for different values of $h$ when $w=1$. 
effect on the wall geometry and the blood flow. Even although the difference between the stenosis development may be negligible between these cases, this explains the small number of points identified in figures 5 (a) and (b) where $\Delta T_{s}^{*}$ is not small. These points do not overturn the view that the simulations with $h=0.1,0.3$ and 0.5 are effectively independent of $h$. Figure 5 (c) shows small, but slightly larger differences between the simulations. This suggests that $h=0.5$ should be used as an upper limit, rather than $h=0.7$. As expected figures $5(\mathrm{~d})$ and (e) highlight the fact that $h=0.9$ and 1.0 are not suitable due to a lack of resolution.

The effect of $w$ on the stenosis development was also considered. This is shown in figure 6 for $w=1$ and $w=2$ for the case $h=0.3$. Figures 6 (a) and (b) depict the evolution in terms of $T_{s}^{*}$ and show a different behaviour for the two cases. These

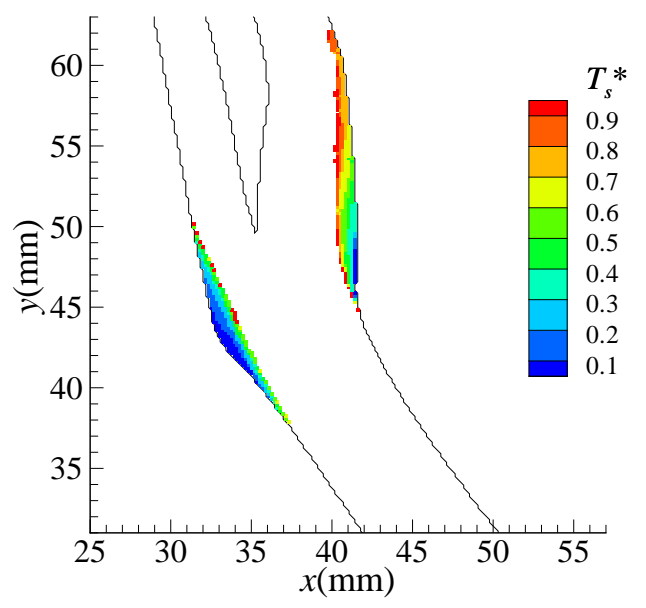

(a) $h=0.3, w=1$

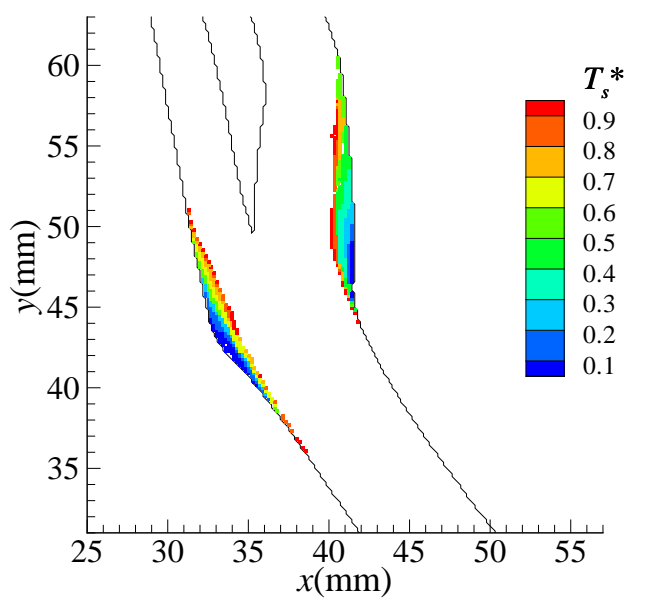

(b) $h=0.3, w=2$

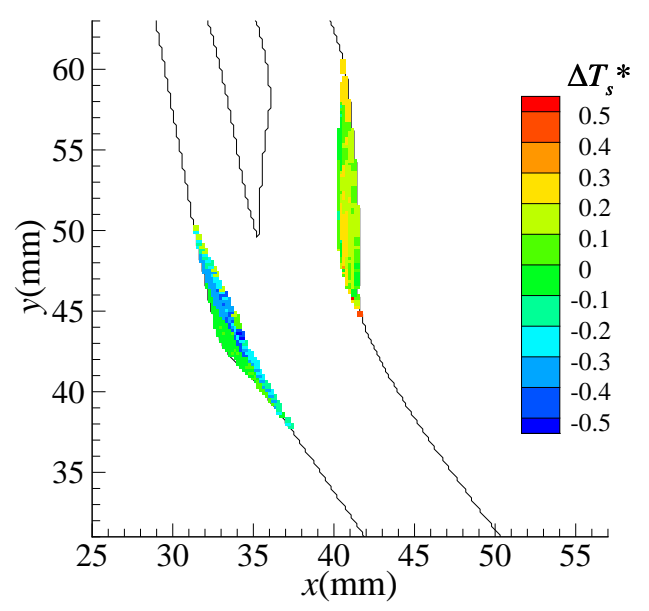

(c) $h=0.3, \Delta T_{s}^{*}=\left.T_{s}^{*}\right|_{w=2}-\left.T_{s}^{*}\right|_{w=1}$

Figure 6. Comparison of $h=0.3$ results for $w=1$ and $w=2$. 
differences are highlighted in figure 6(c) where the differences can be related to the layered growth occurring at different rates. This behaviour is similar to that observed for the larger values of $h$ and suggests that the resolution is not sufficient for $w=2$ and that the model should be run with $w=1$.

The effect of the stenosis growth on the blood flow is shown in figures 7 and 8 at the peak flow and during the deceleration phase respectively. The images on the

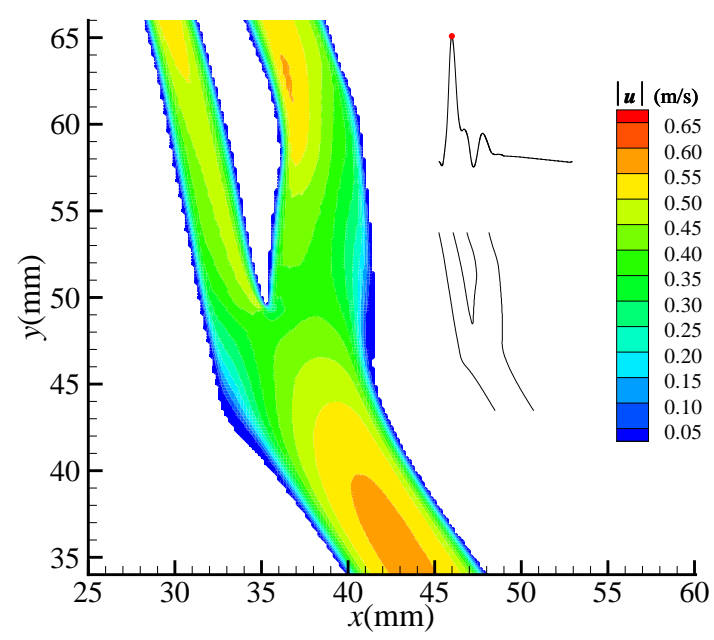

(a) Healthy artery velocity field

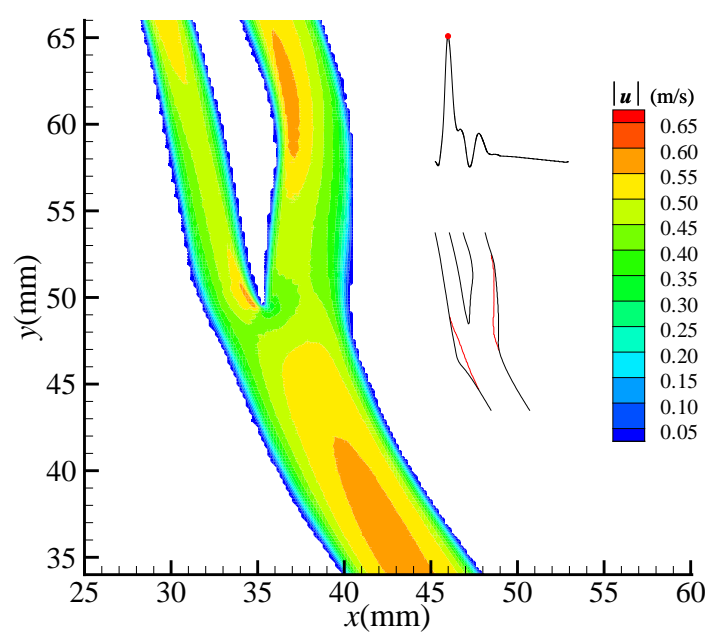

(b) Stenosed artery velocity field

Figure 7. Velocity field for the healthy artery geometry (left) and the stenosed geometry corresponding to $T_{s}^{*}=1$ (right) at peak flow. The driving pulse and the healthy geometry are also shown as an insert in each figure, while the pulse phase and the stenosed geometry are depicted in the inserts by the red dot and the red geometry respectively.

left are for the healthy artery and the ones on the right are for the stenosed geometry at $T_{s}^{*}=1$. Figure 7 depicts the velocity field at the peak in the cardiac cycle. The stenosed geometry shows a higher velocity through both the internal and external carotid branches when compared to the healthy geometry, similar to the 'high velocity jet' observed in the Doppler measurements of [47], as well as the PIV measurements of [48]. Figure 8 shows significant vorticity developing in the healthy artery towards the bottom of both the internal and external carotid arteries. For the stenosed geometry this is no longer present. This is consistent with the MRI images and CFD results reported by [49] It should be noted that [47-49] present results for a phantom; however, these were based on patient measurements and so provide a realistic geometry for a stenosed artery. Additionally, the authors considered a more severe stenosis on only one wall of the artery. The more severe stenosis results in an increased narrowing of the artery and so the jet nature of the flow is more pronounced. This leads to vorticity/oscillations further from the bifurcation, which are not present here. Despite these differences, and the natural differences between individual artery geometries, the similarities suggest that the stenosis development model is predicting a realistic geometry for the stenosed 


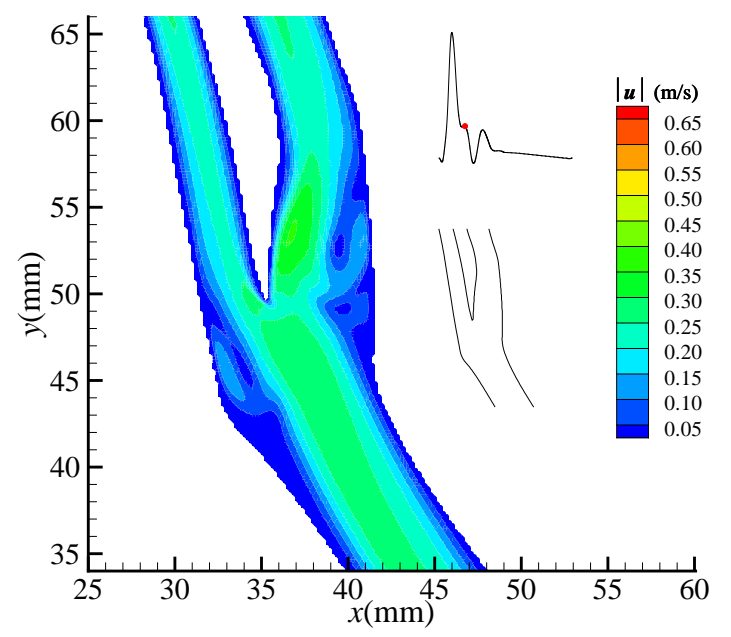

(a) Healthy artery velocity field

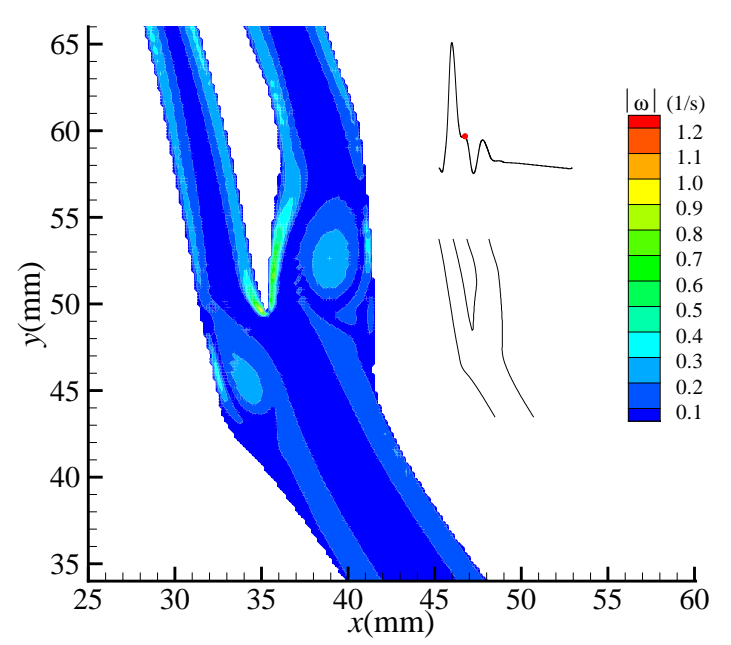

(c) Healthy artery vorticity field

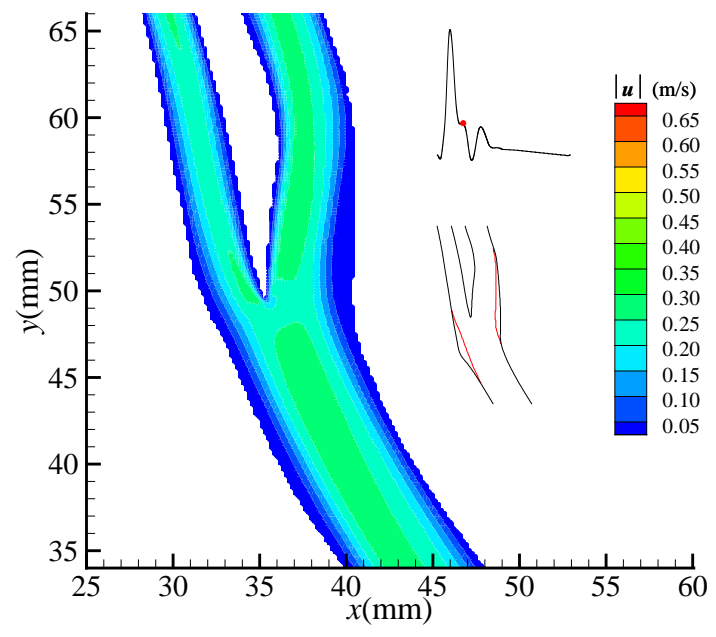

(b) Stenosed artery velocity field

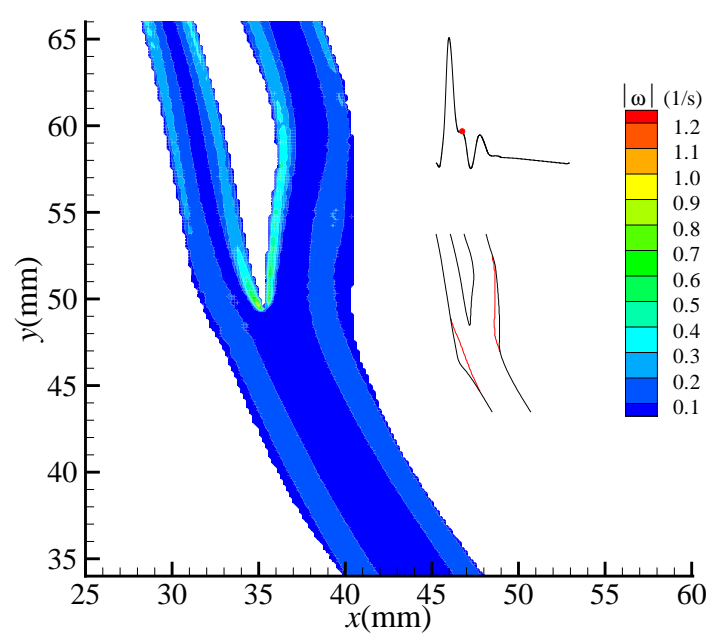

(d) Stenosed artery vorticity field

Figure 8. Velocity (a and b) and vorticity, $\omega$, (c and d) for the healthy artery geometry (left column) and the stenosed geometry corresponding to $T_{s}^{*}=1$ (right column) during the deceleration phase of the cardiac pulse. The driving pulse and the healthy geometry are also shown as an insert in each figure, while the pulse phase and the stenosed geometry are depicted in the inserts by the red dot and the red geometry respectively.

artery. Details of the velocity and vorticity fields over the whole cycle are provided in the supplemental material.

A major concern, even for a relatively low level of stenosis, is that plaque rupture will occur. This is known to occur in regions of high WSS [50], thus it is interesting to examine how the WSS in the stenosed region changes at the stenosis develops. The WSS is found at each boundary point and at each time, using equation (16), as the 
magnitude of $\boldsymbol{\tau}_{w}$ where [42]

$$
\tau_{w \alpha}=2 \nu \rho\left(S_{j \alpha \beta} N_{j \beta}-S_{j \beta \gamma} N_{j \beta} N_{j \gamma} N_{j \alpha}\right),
$$

$S_{j \alpha \beta}$ is found from equation (16) at $\boldsymbol{b}_{j}$ and $\boldsymbol{N}_{j}$ is the normal to the boundary. Although the grid-based normal $\boldsymbol{n}_{j}$ is appropriate for extrapolating $S_{\alpha \beta}$ from the computational nodes to the boundary points, the normal used to find the components in equation (18) was based on the local wall geometry as $\boldsymbol{N}_{j} \cdot \boldsymbol{B}_{j}=0$, where $\boldsymbol{B}_{j}=\boldsymbol{b}_{j+10}-\boldsymbol{b}_{j-10}$ is a measure of the local surface. The value of 10 was selected to give a smooth measure of the surface, and is comparable to the value used in [41].

Figure 9 shows the Peak WSS (PWSS) on the stenosed regions of the outer ECA and ICA walls. In both cases the WSS is low initially as the stenosis starts to develop.

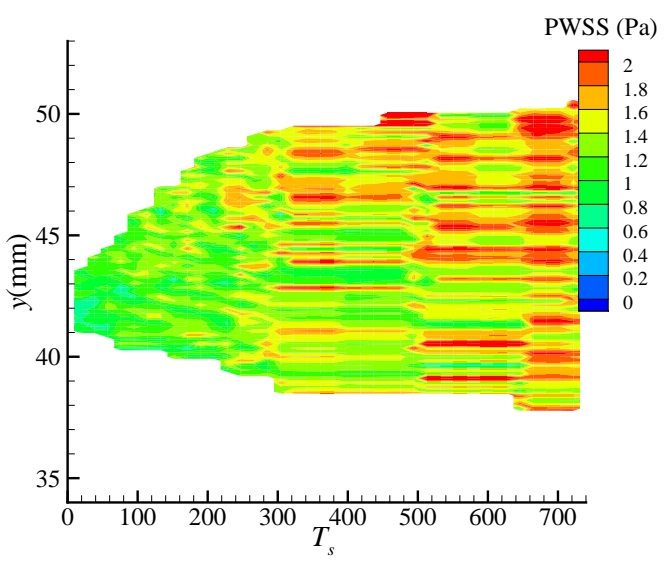

(a) ECA

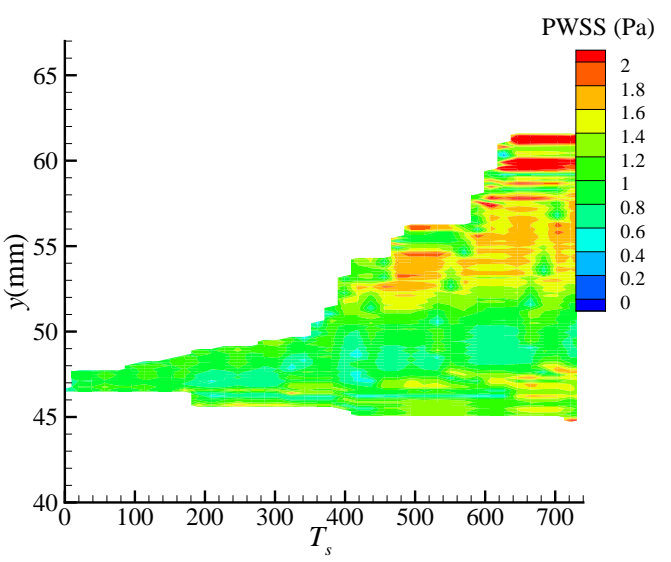

(b) ICA

Figure 9. Peak WSS in the stenosed region of the outer wall of (a) the ECA and (b) the ICA.

As the stenosis develops there is an increase on the PWSS on both walls. Thus as the stenosis develops the wall forces, responsible for plaque rupture, are seen to increase. This is particularly evident in figure 9 (b) for $y>50 \mathrm{~mm}$, where the growth extends well into the ICA. Although there is a significant increase in the PWSS as the stenosis forms, this is in a region where the WSS was originally low, and the values are still within the normal range observed within a healthy artery, suggesting that the chances of rupture at this stage of development are not high.

\section{Conclusions}

A numerical method has been presented for the development of a stenosis in a carotid artery based on the blood flow. The model uses parameters $h$ and $w$ describing the local height and width by which the stenosis develops in each phase (corresponding to a simulation period). The results were seen to converge for $w=1$ and $h \leq 0.5$ 
indicating a range whereby the stenosis can be modelled independently of these nonphysical parameters. Simulations run within this range showed the stenosis development and the changing flow patterns to be consistent with those observed elsewhere. This suggests that the proposed model provides a realistic simulation of the early stages of stenosis development, enabling both the stenosis geometry and the associated blood flow to be studied. Additionally, it suggests that stenosis development in the carotid artery, which has been observed to occur in regions of low velocity and WSS, is in fact related to the haemodynamics and not simply occurring in this region by coincidence. The model has been applied to investigate how the developing stenosis affects the blood flow in terms of the velocity and vorticity in the artery and the PWSS in the stenosed region.

\section{References}

[1] Ala Alwan. Global status report on noncommunicable diseases 2010. World Health Organization, 2011.

[2] Agostino Gnasso, Concetta Irace, Claudio Carallo, Maria Serena De Franceschi, Corradino Motti, Pier Luigi Mattioli, and Arturo Pujia. In vivo association between low wall shear stress and plaque in subjects with asymmetrical carotid atherosclerosis. Stroke, 28(5):993-998, 1997.

[3] Peter H Stone, Ahmet U Coskun, Scott Kinlay, Maureen E Clark, Milan Sonka, Andreas Wahle, Olusegun J Ilegbusi, Yerem Yeghiazarians, Jeffrey J Popma, John Orav, et al. Effect of endothelial shear stress on the progression of coronary artery disease, vascular remodeling, and in-stent restenosis in humans in vivo 6-month follow-up study. Circulation, 108(4):438-444, 2003.

[4] Yiannis S. Chatzizisis, Ahmet Umit Coskun, Michael Jonas, Elazer R. Edelman, Charles L. Feldman, and Peter H. Stone. Role of endothelial shear stress in the natural history of coronary atherosclerosis and vascular remodelingmolecular, cellular, and vascular behavior. Journal of the American College of Cardiology, 49(25):2379-2393, 2007.

[5] Yiannis S Chatzizisis, Ahmet U Coskun, Michael Jonas, Elazer R Edelman, Peter H Stone, and Charles L Feldman. Risk stratification of individual coronary lesions using local endothelial shear stress: a new paradigm for managing coronary artery disease. Current opinion in cardiology, $22(6): 552-564,2007$.

[6] Colin G Caro. Discovery of the role of wall shear in atherosclerosis. Arteriosclerosis, thrombosis, and vascular biology, 29(2):158-161, 2009.

[7] L Goubergrits, K Affeld, J Fernandez-Britto, and L Falcon. Atherosclerosis and flow in carotid arteries with authentic geometries. Biorheology, 39(3-4):519-524, 2001.

[8] C K Zarins, D P Giddens, B K Bharadvaj, V S Sottiurai, R F Mabon, and S Glagov. Carotid bifurcation atherosclerosis. quantitative correlation of plaque localization with flow velocity profiles and wall shear stress. Circulation Research, 53(4):502-514, 1983.

[9] David N Ku, Don P Giddens, Christopher K Zarins, and Seymour Glagov. Pulsatile flow and atherosclerosis in the human carotid bifurcation. positive correlation between plaque location and low oscillating shear stress. Arteriosclerosis, thrombosis, and vascular biology, 5(3):293-302, 1985 .

[10] Caroline Cheng, Dennie Tempel, Rien van Haperen, Arjen van der Baan, Frank Grosveld, Mat JAP Daemen, Rob Krams, and Rini de Crom. Atherosclerotic lesion size and vulnerability are determined by patterns of fluid shear stress. Circulation, 113(23):2744-2753, 2006.

[11] James M. Buick, M. Atig, D. J. Skulina, D. M. Campbell, J. P. Dalmont, and J. Gilbert. Investigation of non-linear acoustic losses at the open end of a tube. The Journal of the Acoustical Society of America, 129(3):1261-1272, 2011. 
[12] Amit Gupta and Ranganathan Kumar. Lattice boltzmann simulation to study multiple bubble dynamics. International Journal of Heat and Mass Transfer, 51(21):5192-5203, 2008.

[13] Irina Ginzburg. A free-surface lattice boltzmann method for modelling the filling of expanding cavities by bingham fluids. Philosophical Transactions of the Royal Society of London A: Mathematical, Physical and Engineering Sciences, 360(1792):453-466, 2002.

[14] C Manwart, U Aaltosalmi, A Koponen, R Hilfer, and J Timonen. Lattice-boltzmann and finitedifference simulations for the permeability for three-dimensional porous media. Physical Review E, 66(1):016702, 2002.

[15] Huabing Li, Haiping Fang, Zhifang Lin, ShiXiong Xu, and Shiyi Chen. Lattice boltzmann simulation on particle suspensions in a two-dimensional symmetric stenotic artery. Physical review E, 69(3):031919, 2004.

[16] J Boyd and J M Buick. Three-dimensional modelling of the human carotid artery using the lattice Boltzmann method: I. Model and velocity analysis. Physics in Medicine and Biology, 53(20):5767-5779, 2008.

[17] Junfeng Zhang, Paul C. Johnson, and Aleksander S. Popel. Red blood cell aggregation and dissociation in shear flows simulated by lattice boltzmann method. Journal of Biomechanics, $41(1): 47-55,2008$.

[18] MM Dupin, I Halliday, CM Care, and LL Munn. Lattice boltzmann modelling of blood cell dynamics. International Journal of Computational Fluid Dynamics, 22(7):481-492, 2008.

[19] B Min Yun, LP Dasi, CK Aidun, and AP Yoganathan. Computational modelling of flow through prosthetic heart valves using the entropic lattice-boltzmann method. Journal of Fluid Mechanics, 743:170-201, 2014.

[20] Masaaki Tamagawa and Sumiaki Matsuo. Predictions of thrombus formation using Lattice Boltzmann Method (modeling of adhesion force for particles to wall). JSME International Journal Series C, 47(4):1027-1034, 2004.

[21] Masaaki Tamagawa, Hiroaki Kaneda, Miki Hiramoto, and Sho Nagahama. Simulation of thrombus formation in shear flows using Lattice Boltzmann Method. Artificial Organs, 33(8):604-610, 2009.

[22] SE Harrison, SM Smith, J Bernsdorf, DR Hose, and PV Lawford. Application and validation of the Lattice Boltzmann Method for modelling flow-related clotting. Journal of Biomechanics, 40(13):3023-3028, 2007.

[23] Jörg Bernsdorf, Sarah E Harrison, Stephen M Smith, Patricia V Lawford, and D Rodney Hose. Applying the Lattice Boltzmann technique to biofluids: A novel approach to simulate blood coagulation. Computers \& Mathematics with Applications, 55(7):1408-1414, 2008.

[24] Gilead Moiseyev and Pinhas Z. Bar-Yoseph. No need for particle tracing: From accumulating fluid properties to novel blood coagulation model in the lattice boltzmann method. Journal of Biomechanics, 43(5):864-870, 2010.

[25] H Karimpour and E Javdan. Simulation of stenosis growth in the carotid artery by Lattice Boltzmann Method. Journal of Mechanics in Medicine and Biology, 14(02), 2014.

[26] Alexandre Masselot and Bastien Chopard. A lattice boltzmann model for particle transport and deposition. EPL (Europhysics Letters), 42(3):259, 1998.

[27] G Gamble, J Zorn, G Sanders, S MacMahon, and N Sharpe. Estimation of arterial stiffness, compliance, and distensibility from m-mode ultrasound measurements of the common carotid artery. Stroke, 25(1):11-6, 1994.

[28] Z. Guo, C. Zheng, and B. Shi. An extrapolation method for boundary conditions in lattice Boltzmann method. Physics of Fluids, 14(6):2007-2010, 2002.

[29] S. Chen and G. D. Doolen. Lattice boltzmann method for fluid flows. Annual Review of Fluid Mechanics, 30:329-364, 1998.

[30] Y. H. Qian, D. d'Humieres, and P. Lallemand. Lattice BGK models for Navier-Stokes equation. Europhysics Letters, 17(6):479-484, 1992.

[31] P. L. Bhatnagar, E. P. Gross, and M. Krook. A model for collision processes in gases. I: small 
amplitude processes in charged and neutral one-component system. Physical Review, 94:511525, 1954.

[32] S. Wolfram. Cellular automaton fluids 1: Basic theory. Journal of Statistical Physics, 45(3/4):471$529,1986$.

[33] U. Frisch, D. d' Humières, B. Hasslacher, P. Lallemand, Y. Pomeau, and J.-P. Rivet. Lattice gas hydrodynamics in two and three dimensions. Complex Systems, 1:649-707, 1987.

[34] Shiyi Chen and Gary D. Doolen. Lattice Boltzmann Method for fluid flows. Annual Review of Fluid Mechanics, 30(1):329-364, 1998.

[35] A. Artoli. Mesoscopic Computational Haemodynamics. Technical report, University of Amsterdam, October 2003.

[36] J. Boyd, J.M. Buick, J.A. Cosgrove, and P. Stansell. Application of the lattice Boltzmann method to arterial flow simulation: Investigation of boundary conditions for complex arterial geometries. Australasian Physical and Engineering Sciences in Medicine, 27(4):147-152, 2004.

[37] J. Boyd, J. Buick, J. A. Cosgrove, and P. Stansell. Application of the lattice Boltzmann model to simulated stenosis growth in a two-dimensional carotid artery. Physics in Medicine and Biology, 50(20):4783-4796, 2005.

[38] D.W. Holdsworth, C.J.D. Norley, R. Frayne, D.A. Steinman, and B.K. Rutt. Characterisation of common carotid artery blood-flow waveforms in normal human subjects. Jounal of Physiological Measurements, 20:219-240, 1999.

[39] M. Neal. A Study of the Brass Instrument Lip Reed Mechanism using Artificial Lips and Lattice Boltzmann Flow Simulations. PhD thesis, University of Edinburgh, Edinburgh, Scotland, 2002.

[40] J Boyd and J M Buick. Comparison of Newtonian and non-Newtonian flows in a two-dimensional carotid artery model using the lattice Boltzmann method. Physics in Medicine and Biology, 52(20):6215-6228, 2007.

[41] Maciej Matyka, Zbigniew Koza, and ukasz Mirosaw. Wall orientation and shear stress in the lattice boltzmann model. Computers \&f Fluids, 73:115 - 123, 2013.

[42] Xiuying Kang. Assessment of the pulsatile wall shear stress in the stenosed and recanalized carotid bifurcations by the lattice boltzmann method. Computers \& Fluids, 97:156 - 163, 2014.

[43] Wolfgang Steinke, Christof Kloetzsch, and Michael Hennerici. Carotid artery disease assessed by color doppler flow imaging: correlation with standard doppler sonography and angiography. American journal of neuroradiology, 11(2):259-266, 1990.

[44] James Dix and James Skrocki. Evaluation of carotid stenosis by angiography: potential bias toward overestimated measurements introduced by prior interpretation of doppler sonograms. American journal of neuroradiology, 21(4):639-642, 2000.

[45] Chun Yuan, William S Kerwin, Vasily L Yarnykh, Jianming Cai, Tobias Saam, Baocheng Chu, Norihide Takaya, Marina S Ferguson, Hunter Underhill, Dongxiang Xu, Fei Liu, and Thomas Hatsukami. Mri of atherosclerosis in clinical trials. NMR in Biomedicine, 19(6):636-654, 2006.

[46] Michele Anzidei, Alessandro Napoli, Beatrice Cavallo Marincola, Miles A Kirchin, Cristina Neira, Daniel Geiger, Fulvio Zaccagna, Carlo Catalano, and Roberto Passariello. High-resolution steady state magnetic resonance angiography of the carotid arteries: are intravascular agents necessary?: feasibility and preliminary experience with gadobenate dimeglumine. Investigative radiology, 44(12):784-792, 2009.

[47] Tamie L Poepping, N Nikolov, N Rankin, Mark Lee, and David W Holdsworth. An in vitro system for Doppler ultrasound flow studies in the stenosed carotid artery bifurcation. Ultrasound in Medicine E Biology, 28(4):495-506, 2002.

[48] David A Steinman, Tamie L Poepping, Mauro Tambasco, Richard N Rankin, and David W Holdsworth. Flow patterns at the stenosed carotid bifurcation: effect of concentric versus eccentric stenosis. Annals of Biomedical Engineering, 28(4):415-423, 2000.

[49] Ian Marshall, Shunzhi Zhao, Panorea Papathanasopoulou, Peter Hoskins, and X Yun Xu. MRI and CFD studies of pulsatile flow in healthy and stenosed carotid bifurcation models. Journal of Biomechanics, 37(5):679-687, 2004. 
[50] CJ Slager, JJ Wentzel, FJH Gijsen, A Thury, AC van der Wal, JA Schaar, and PW Serruys. The role of shear stress in the destabilization of vulnerable plaques and related therapeutic implications. Nature Clinical Practice Cardiovascular Medicine, 2(9):456-464, 2005. 